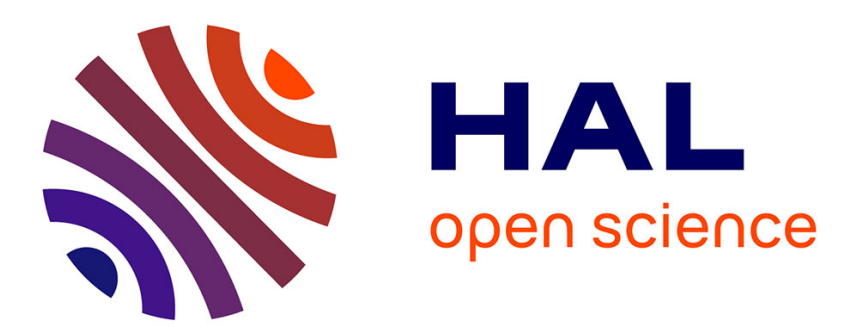

\title{
Les datations par traces de fission de l'Uranium. Principes et applications aux problèmes du Quaternaire. \\ Gérard Poupeau
}

\section{To cite this version:}

Gérard Poupeau. Les datations par traces de fission de l'Uranium. Principes et applications aux problèmes du Quaternaire.. Bulletin de l'Association française pour l'étude du quaternaire, 1979, 16 (1), pp.15-26. 10.3406/quate.1979.1342 . hal-03409224

\section{HAL Id: hal-03409224 \\ https://hal.science/hal-03409224}

Submitted on 29 Oct 2021

HAL is a multi-disciplinary open access archive for the deposit and dissemination of scientific research documents, whether they are published or not. The documents may come from teaching and research institutions in France or abroad, or from public or private research centers.
L'archive ouverte pluridisciplinaire HAL, est destinée au dépôt et à la diffusion de documents scientifiques de niveau recherche, publiés ou non, émanant des établissements d'enseignement et de recherche français ou étrangers, des laboratoires publics ou privés. 


\section{Les datations par traces de fission de l'Uranium. Principes et applications aux problèmes du Quaternaire.}

\section{Gérard Poupeau}

\section{Résumé}

La méthode des traces de fission est particulièrement bien adaptée à la datation de certains types d'événements au sein du quaternaire. Après une présentation des aspects théoriques de cette méthode, on propose une revue de ses applications aux problèmes qui intéressent les quaternaristes : volcanisme et stratigraphie, tectonique récente, paléomagnétisme, applications en archéologie et à la datation des fossiles et artefacts humains les plus anciens.

\section{Citer ce document / Cite this document :}

Poupeau Gérard. Les datations par traces de fission de I'Uranium. Principes et applications aux problèmes du Quaternaire.. In: Bulletin de l'Association française pour l'étude du quaternaire, vol. 16, n¹-2, 1979. pp. 15-26;

doi : https://doi.org/10.3406/quate.1979.1342

https://www.persee.fr/doc/quate_0004-5500_1979_num_16_1_1342

Fichier pdf généré le 06/11/2020 


\section{LES DATATIONS PAR TRACES DE FISSION DE L'URANIUM. PRINCIPES ET APPLICATIONS AUX PROBLÈMES DU QUATERNAIRE \\ par G. POUPEAU*}

\section{RESUME}

La méthode des traces de fission est particulièrement bien adaptée à la datation de certains types d'évènements au sein du quaternaire. Après une présentation des aspects théoriques de cette méthode, on propose une revue de ses applications aux problèmes qui intéressent les quaternaristes : volcanisme et stratigraphie, tectonique récente, paléomagnétisme, applications en archéologie et à la datation des fossiles et artefacts humains les plus anciens.

\section{I. - INTRODUCTION}

La fission spontanée de ${ }^{238} \mathrm{U}$ a été découverte en 1940 (Petrzhak et Flerov), mais ce n'est que dans les années 60.70 que ce chronomètre nucléaire fut appliqué avec succès en géochronologie. Il existe deux façons d'utiliser la fission pour dater une roche. Soit on mesure les isotopes produits par la fission d'un noyau d'uranium 238 : c'est la méthode ${ }^{238} \mathrm{U}-\mathrm{Xe}$ (Shukoljukov et al., 1974 ; Teitsma et al., 1975). Soit on détecte physiquement, dans le matériau à dater, les évènements individuels de fission, dans la méthode des traces proposée par Price et Walker (1963 b).

Les traces de fission spontanée de l'uranium avaient été observées pour la première fois par Price et Walker en 1962. Les premières datations par la méthode des traces datent de 1964 (Fleischer et al, 1964 a ; Maurette et al, 1964). Depuis, cette méthode a connu un développement rapide et montré une grande souplesse d'utilisation puisqu'en effet elle s'adapte aussi bien à la datation de matériaux geologiques (Fleischer et al, 1971 ; Bigazzi et Bonadonna, 1973) et archéologiques (Fleischer, 1976) très récents, qu'à l'analyse des évènements de fission enregistrés dans les roches les plus anciennes du système solaire (Drozd et al, 1977).
Le but de cet article est de présenter d'une part la théorie de la méthode des traces de fission ; d'autre part, une revue de ses applications aux problèmes du quaternaire. Pour un traitement plus détaillé de la méthode, nous renvoyons à Fleischer et Hart (1972) et Fleischer et al, (1975).

\section{II. - THEORIE}

La datation par traces de fission est possible parce que dans certains solides, les fragments de fission de l'uranium produisent une zone relativement stable de dégâts le long de leurs parcours.

La fission d'un atome d'uranium produit deux noyaux-fils animés d'une énergie cinétique de l'ordre de 0.5 à $1 \mathrm{MeV} /$ nucléon, qui sont éjectés selón des directions opposées. Dans un solide isolant, ces noyaux, vont produire une zone linéaire de dommages intenses. Le mécanisme de formation de cette zone perturbée, ou trace latente, est illustré dans la figure 1. Les fragments de fission, qui sont des noyaux fortement ionisés, interagissent le long de leur parcours avec les électrons du milieu, et créent une zone chargé positive- 

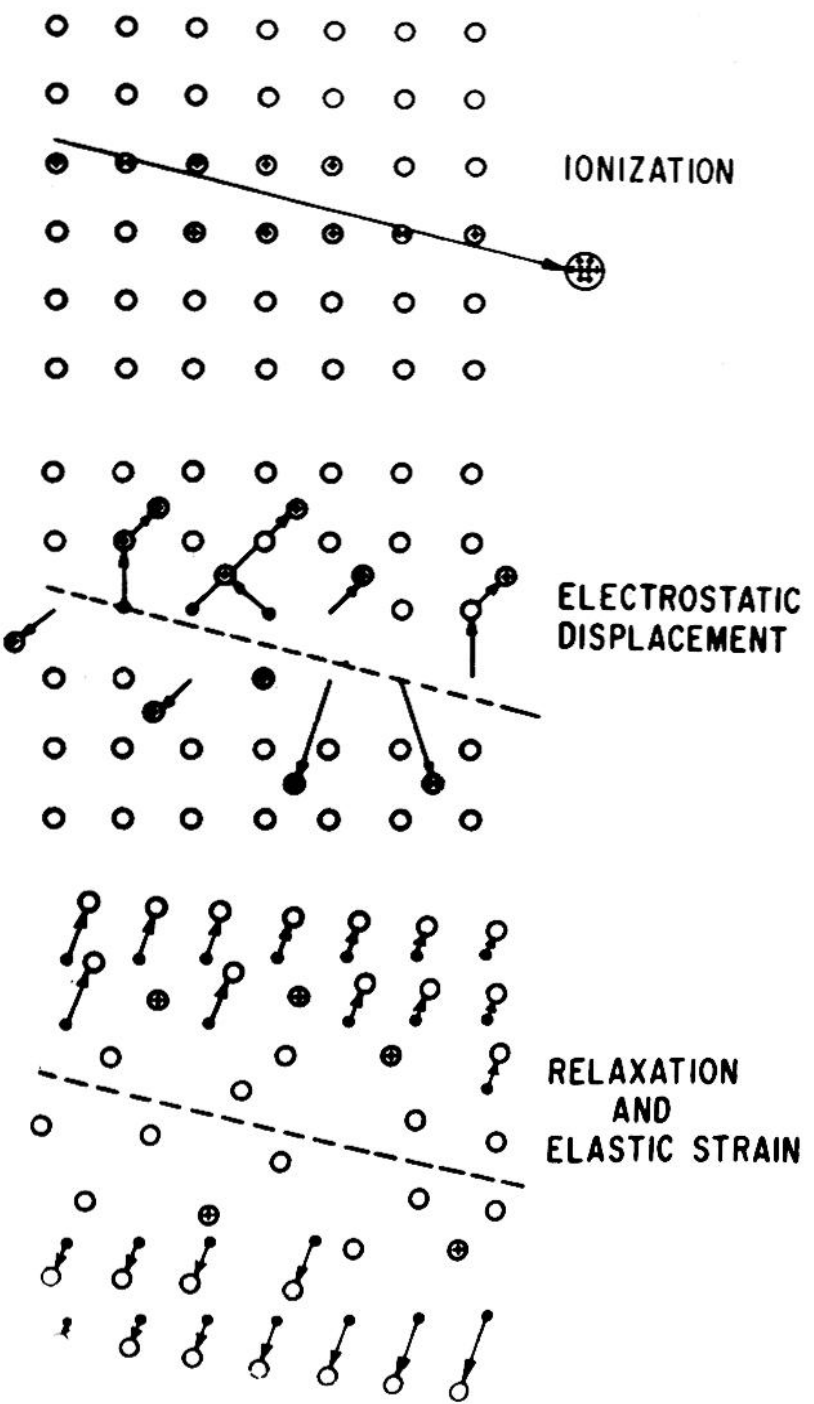

Fig. 1. - Formation de la trace latente dans un solide isolant non organique selon le modèle de "pic d'explosion ionique" (Fleischer et al, 1965 e). La particule chargée initiale ionise les atomes le long de sa trajectoire (haut). Les ions positifs ainsi formés vont se trouver éjectés par répulsion électrostatique dans le solide en position d'intersticiels créant ainsi des vacances (milieu). La région perturbée se rééquilibre, exerçant une contrainte sur le réseau (bas) ; d'après Fleischer et al., 1975). C'est l'existence de ces distorsions du réseau au niveau de la trace latente qui rendent possible son observation en microscopie électronique.

ment. La formation de la trace latente résulterait alors, secondairement, de l'éjection (par répulsion électrostatique) en position d'intersticiels, des ions positifs produits au coeur de la trace. Dans un solide non isolant, les traces ne peuvent se former en raison de la présence d'électrons libres venant compenser aussitôt après le passage des fragments de fission le déficit en charges négatives créé par ionisation le long de leur parcours. C'est pour cette raison que l'on ne peut révéler de traces dans les métaux, ou certains semi-conducteurs.

Les traces latentes présentent, selon les minéraux, une longueur de 10 à 20 microns. Le noyau d'uranium initial se trouvait avant la fission au voisinage du centre de la trace. Le diamètre des traces latentes, tel qu'il apparaît en microscopie electronique n'est que de quelques dizaines d'angstroems. Price et Walker (1962 b) ont montré qu'il était possible de rendre ces traces visibles en microscopie optique au moyen d'une attaque chimique appropriée, agrandissant leur diamètre jusqu'à une fraction de micron (figure 2 ).

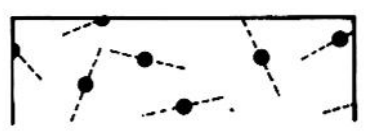

a

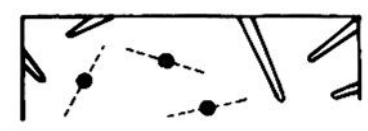

b
Fig. 2. - Traces de fission latentes et traces révélées. A gauche, traces latentes; le point central indique la position de l'atomepère d'uranium. Le diamètre mesurable de ces traces en $\mathrm{mi}$ croscopie électronique est de l'ordre de quelques dizaines d'augstroems. Celles qui intersectent une surface libre du détecteur peuvent être "rúvélées" par une attaque chimique préférenticlle (à droite). L'accroissement de leur diamètre jusque vers $\sim 1$ micron les rend visibles en microscopie optique.

Ce sont les traces ainsi révélées que l'on utilise pour les datations (figure 3).

Dans les minéraux terrestres, la seule source de traces est la fission spontanée et éventuellement induite de l'uranium et du thorium (Price et Walker, 1963 b). La proportion de fissions induites est géneralement négligeable, sauf en présence d'une fluence anormalement élevée de neutrons, telle qu'on en rencontre dans les gisements d'uranium (Price et Walker, $1963 \mathrm{~b}$; Petit, 1977) ou a proximité d'un site d'explosion nucléaire (Walker, 1963). II existe trois isotopes naturels qui fissionnent spontanément : ${ }^{232} \mathrm{Th},{ }^{235} \mathrm{U}$ et ${ }^{238} \mathrm{U}$. Seul ce dernier fissionne avec une période suffisamment courte pour donner un nombre non négligeable de traces de fission spontanée. En pratique donc, toutes les traces de fission révélées dans les minéraux terrestres doivent être attribuées à la fission spontanée de ${ }^{238} \mathrm{U}$. De même que ${ }^{232}$ Th et ${ }^{235} \mathrm{U},{ }^{238} \mathrm{U}$ décroît également par radioactivité $\alpha$. II produit plusieurs millions de désintégrations $\alpha$ pour un évènement de fission.

\section{L'équation d'âge}

La densité (nombre par unité de surface) de traces de fission spontanée dans le matériel à dater est une fonction de son âge et de sa teneur en uranium. La détermination d'un âge par traces de fission nécessite la mesure de la densité de traces fossiles $\rho_{F}$ et de la teneur en uranium. Pour une roche d'âge $<10^{8}$ ans, la densité de traces fossiles de ${ }^{238} \mathrm{U}$ est donné par la relation :

$$
\rho_{F}=f_{238} N C^{238} R^{238} \lambda_{F} t
$$



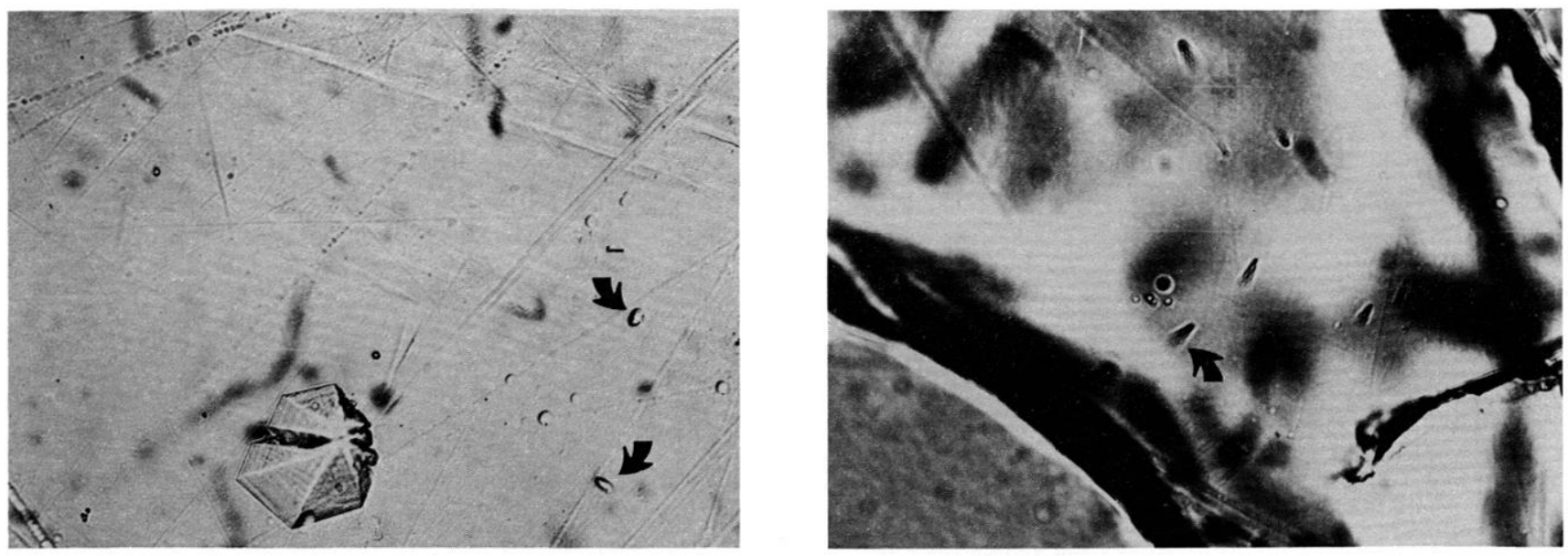

a

b

Fïg. 3. - Traces de fission de l'uranium dans différents détecteurs naturels : obsidienne (a), apatite (b). La longueur maximum des traces varie de $\sim 8$ microns (obsidienne) à 18 microns (apatite).

où

$$
\begin{aligned}
f_{238}= & \text { efficacité de révélation des traces de }{ }^{238} \mathrm{U} \\
\mathrm{N}= & \text { nombre d'atomes par unité de volume du ma- } \\
& \text { tériau } \\
\mathrm{C}^{238}= & \text { concentration (atome/atome) en }{ }^{238} \mathrm{U} \\
\mathrm{R}^{238}= & \text { longueur révélable des traces de fission spon- } \\
& \text { tanée de }{ }^{238} \mathrm{U} \\
= & \text { temps depuis lequel le matériau a commencé } \\
& \text { à enregistrer les traces. C'est l'“âge" du maté- } \\
& \text { riau. } \\
\lambda_{F}= & \text { constante de décroissance par fission sponta- } \\
& \text { née. }
\end{aligned}
$$

L'uranium 235 pouvant fissionner sous l'influence des neutrons thermiques, on détermine la teneur en ${ }^{235} \mathrm{U}$ dans le matériau à dater au moyen d'une exposition dans un réacteur nucléaire. La densité $\rho_{\mathrm{i}}$ de traces de fission induite de ${ }^{235} \mathrm{U}$ est égale à :

$$
\rho_{\mathrm{i}}=\mathrm{f}_{235} \mathrm{NC}^{235} \mathrm{R}^{235} \sigma \phi
$$

où

$$
\begin{aligned}
\sigma= & \text { section efficace de fission de }{ }^{235} \mathrm{U} \text { par les } \\
& \text { neutrons thermiques } \\
\boldsymbol{\Psi}= & \text { dose (neutrons } / \mathrm{cm}^{2} \text { ) de neutrons thermiques }
\end{aligned}
$$

Les autres quantités sont définies comme précédemment.

Dans les datations par traces de fission, on mesure la dose de neutrons au moyen d'un verre calibré ayant une teneur bien connue en uranium, situé pendant l'irradiation, au contact de l'échantillon à dater. La densité $\rho_{n}$ de traces de fission induite mesurée dans ce verre ou dans un détecteur auxiliaire (mica par exemple) appliqué contre ce verre pendant l'irradiation vaut :

$$
\rho_{\mathrm{n}}=\mathrm{K} \Phi
$$

où $\mathrm{K}$ est une constante déterminée expérimentalement (voir plus loin).
En combinant (1), (2) et (3) on obtient pour équation d'âge l'expression :

$$
\left\{\begin{array}{l}
\mathrm{t}=\mathrm{A} \frac{\rho_{\mathrm{F}}}{\rho_{\mathrm{i}}} \rho_{\mathrm{n}} \\
\mathrm{A}=\frac{\mathrm{f}_{235}}{\mathrm{f}_{238}} \frac{\mathrm{R}^{235}}{\mathrm{R}^{238}} \frac{\sigma \mathrm{I}}{\mathrm{K} \lambda_{\mathrm{F}}}
\end{array}\right.
$$

où I est le rapport des abondances naturelles ${ }^{235} \mathrm{U} /$ ${ }^{238} \mathrm{U}$.

On admet généralement que $f_{235}=f_{238}$

D'autre part, lorsque aucun recuit thermique n'a affecté les traces, $R^{235}=R^{238}$. Dans ce cas l'équation d'âge se simplifie et devient :

$$
\mathrm{t}=\frac{\rho_{\mathrm{F}}}{\rho_{\mathrm{i}}} \rho_{\mathrm{n}} \cdot \frac{\sigma \mathrm{I}}{\mathrm{K} \lambda_{\mathrm{F}}}=\mathrm{A}^{\prime} \frac{\rho_{\mathrm{F}}}{\rho_{\mathrm{i}}} \rho_{\mathrm{n}}
$$

où

$$
\begin{aligned}
& \sigma=582 \times 10^{-24} \mathrm{~cm}^{-2} \\
& I=7,26 \times 10^{-3}
\end{aligned}
$$

Les nombreuses déterminations de $\lambda_{F}$ proposées depuis près de 40 ans oscillent entre $\lambda_{F}=0,7 \times 10^{-17}$ ans $^{-1}$ (Petrzhak et Flerov, 1940) et $\lambda_{F}=12 \times 10^{-17}$ ans $^{-1}$ (Gerling et al, 1959). Il n'existe actuellement pas de consensus parmi les utilisateurs de la méthode des traces quant au choix de la constante de fission $\lambda_{F}$. Deux valeurs principalement de celle-ci sont en usage. Il s'agit respectivement de celle déterminée par Fleischer et Price (1964 a) $\lambda_{F}=6,85 \pm 0,20 \times 10^{-17}$ ans $^{-1}$ et Galliker et al $(1970) \lambda_{F}=8,46 \pm 0,06 \times 10^{-17}$ ans $^{-1}$.

La méthode des traces permet en principe de déterminer la valeur de $\lambda_{\mathrm{F}}$ à employer. On peut en effet résoudre l'équation (5) pour $\lambda_{F}$ en utilisant des échantillons d'âge connu ou un détecteur placé pendant un 
temps connu contre une feuille d'uranium. Dans ce dernier cas, on peut aussi calculer $\lambda_{F}$ d'après l'équation (1), (Roberts et al, 1968).

La première détermination de $\lambda_{F}$ à partir de l'équation (5) est celle de Fleischer et Price. Toutes les déterminations ultérieures de $\lambda_{F}$ faites avec la méthode des traces sur des phases cristallines de roches déjà datées ou à partir de feuille d'uranium donnent des résultats assez proche de la valeur de Fleischer et Price (voir références dans Fleischer et al, 1975 ; Izett et Naeser, 1976 ; Hurford et Gleadow, 1977). La valeur $\lambda_{F}=6,85 \times 10^{-17} \mathrm{ans}^{-1}$ est la plus généralement utilisée dans la géochronologie par traces de fission.

La valeur de $\lambda_{F}$ que l'on obtient à partir de l'équation (5) en utilisant des verres (fabriqués en volcaniques) est nettement plus élevée, de l'ordre de $8,5 \times 10^{-17}$ ans $^{-1}$ (Storzer, 1970 ; Storzer et Poupeau, 1974 ; Wagner et al. 1975 ; Thiel et Herr, 1976). Certains auteurs (Wagner et al. 1976) utilisent donc comme $\lambda_{F}$ la valeur $\lambda_{F}=8,46 \pm 0,04 \times 10^{-17}$ ans $^{-1}$ obtenue avec une chambre à bulle rotative déterminée avec une meilleure précision que par la méthode des traces.

Le fait que l'emploi de l'une ou l'autre des deux valeurs de $\lambda_{F}$ les plus fréquemment employées puisse produire des âges traces de fission concordants avec les âges $\mathrm{K}-\mathrm{Ar}$ de roches volcaniques peut résulter de différences systématiques de calibration entre différents groupes. Il est également possible que verres et minéraux aient un comportement différent vis-àvis des traitements auxquels ils sont soumis dans le processus de détermination d'un âge par traces de fission.

\section{Mesure du flux de neutrons}

La mesure du flux de neutrons est assurée par un verre moniteur calibré. La teneur en uranium de ce moniteur doit être adaptée à la dose de neutrons à mesurer, et sa distribution doit être la plus uniforme possible. Des verres standards, préparés par Carpenter et Reimer (1974) sont disponibles au U.S. National Bureau of Standards. Ces verres, présentés sous forme de pastilles, sont utilisés par plusieurs groupes. Livrés conjointement avec des pastilles du même verre déjà irradiées avec une dose connue de neutrons, ils permettent une calibration par comparaison. Il suffit en effet pour calibrer un verre standard, de comparer la densité de traces induites dans ce verre au cours d'une irradiation à celle mesurée dans le verre N.B.S. irradié avec une dose connue. Les traces dans ces deux verres étant révélées dans les mêmes conditions, la dose de neutrons à déterminer est donnée par :

$$
\Phi_{x}=\frac{\rho_{x}}{\rho_{m}} \Phi_{m}
$$

où

$\mathbf{x}=$ dose à déterminer

$\Phi_{m}=$ dose donnée de l'irradiation N.B.S

$\rho_{m}=$ densité de traces dans le moniteur irradié par le N.B.S

$\rho_{\mathrm{x}}=$ densité de traces dans le moniteur utilisé dans l'irradiation $\mathbf{x}$

Il est recommandé toutefois de procéder soi-même à une recalibration de ces standards avec un moniteur d'or ou de cobalt dont la mesure de l'activité induite lors d'une irradiation avec un verre donne une mesure directe de la dose. On détermine ainsi directement le facteur $\mathrm{K}$ de l'équation (3).

Les standards du N.B.S. sont les plus largement utilisés. Deux autres séries sont actuellement disponibles, auprès de, respectivement : D.H. Brill, Corning Museum of Glass, Corning, N.Y. (Schreurs et al, 1971) et R.L. Fleischer, General Electric Laboratory, Schenectady, N.Y. (Fleischer et al, 1965 b).

Les procédures décrites ci-dessus supposent que l'on détermine un âge traces de fission à partir des équations (4) ou (5), en calculant les expressions $A$ ou $A^{\prime}$ terme à terme. Le calcul direct de l'expression $\mathrm{A}$ ou $A^{\prime}$ en utilisant une roche d'âge connu simplifie la procédure de datation en éliminant les problèmes liés au choix de $\lambda_{F}$ et à la calibration de la mesure des flux de neutrons. Dans ce cas si l'on irradie ensemble un matériau d'âge connu $t_{s}$ et le matériau à dater, d'âge $t_{x}$ :

$$
\begin{aligned}
& t_{s}=\left(\frac{\rho_{F}}{\rho_{i}}\right)_{s} \frac{\sigma I \Phi}{\lambda} \\
& t_{x}=\left(\frac{\rho_{F}}{\rho_{i}}\right)_{x} \frac{\sigma I \Phi}{\lambda}
\end{aligned}
$$

D'où l'on tire

$$
\mathrm{t}_{\mathrm{x}}=\left(\frac{\rho_{\mathrm{F}}}{\rho_{\mathrm{i}}}\right) \cdot\left(\frac{\rho_{\mathrm{i}}}{\rho_{\mathrm{F}}}\right) \cdot \mathrm{t}_{\mathrm{s}}
$$

Cette procédure suppose que l'on dispose de roches standard d'âge connu indépendamment. Les roches volcaniques ayant refroidi rapidement après leur mise en place et n'ayant subi aucun réchauffement par la suite apparaissent comme les meilleures candidates. L'un des standards les plus utilisés consiste en un lot de cristaux d'apatites et de zircons du Fish Canyon (Colorado), très bien daté par la méthode potassiumargon, où biotite, homblende, plagioclase et sanidine donnent des âges concordants à $27.2 \pm 0.7$ millions d'années (Steven et al, 1967). Ces standards sont disponibles auprès de C.W. Naeser, U.S. Geological Survey, Denver, Colorado. 


\section{Stabilité des traces}

La détermination d'un âge par la méthode des traces suppose que ces défauts soient relativement stables. En fait, comme les autres chronomètres géologiques nucléaires, les traces sont sensibles aux conditions d'environnement, et tout particulièrement aux effets thermiques (Fleischer et al, 1975).

Les autres effets d'environnement : pression, altérations chimique, etc., n'ont pas fait jusqu'à présent l'objet d'études systématiques. On sait cependant que dans les conditions normales d'emploi de la méthode, des minéraux comme le zircon, l'olivine et l'apatite sont relativement indifférents aux effets de pression hydrostatique (Fleischer et al, 1965 a ; Naeser et Faul, 1969). Par contre, les conditions de stabilité des traces dans des phases telles que verres volcaniques et micas semblent modifiées en présence d'eau sous pression (Lakatos et Miller, 1970, 1972). Enfin, les effets de choc peuvent faire disparaître les traces dans les silicates (Ahrens et al, 1970, Fleischer et al, 1974), ainsi que, peut-être, les pressions orientées (micas, voir Fleischer et al, 1965 a).

Les seules données que l'on possède actuellement sur les effets d'altération sont celles de Gleadow et Lovering (1974). Ces auteurs ont étudié le comportement des apatites, sphènes et zircons d'un même massif granitique dans des échantillons présentant des degrés variables d'altération. Il a été montré que même avec un degré d'altération extrême (argile résiduelle) l'âge trace de fission des sphènes et zircons restait le même que dans la roche saine, alors qu'il pouvait se trouver diminue de $17 \%$ (à la fois par une perte de traces de fission spontanée et de ${ }^{238} \mathrm{U}$ ) dans les apatites.

Des expériences de laboratoire ont montré qu'un recuit thermique, même modéré, peut altérer sensiblement la révélation des traces, voire rendre leur révélation impossible. Cet effacement des traces se produit lorsque les atomes déplacés lors du passage d'un fragment de fission acquièrent suffisamment d'énergie pour rétrodiffuser en direction du coeur de la trace et éventuellement rétablir certaines liaisons chimiques rompues.

Pour des conditions de révélation données, le temps nécessaire $\tau$ pour recuire une fraction donnée de la densité de traces est exprimé par la relation:

$$
\tau=\mathbf{a} \exp (\mathrm{E} / \mathrm{kT})
$$

où

$$
\begin{aligned}
& \mathbf{a}=\text { constante } \\
& \mathbf{E}=\text { énergie d'activation } \\
& \mathbf{k}=\text { constante de Boltzmann } \\
& \mathrm{T}=\text { température absolue }
\end{aligned}
$$

La figure 4 présente les courbes de recuit d'une apatite naturelle pour trois taux de recuits différents. On constate qu'à $275^{\circ} \mathrm{C}$, il suffit d'environ 1 heure pour commencer à affecter la densité de traces révélable

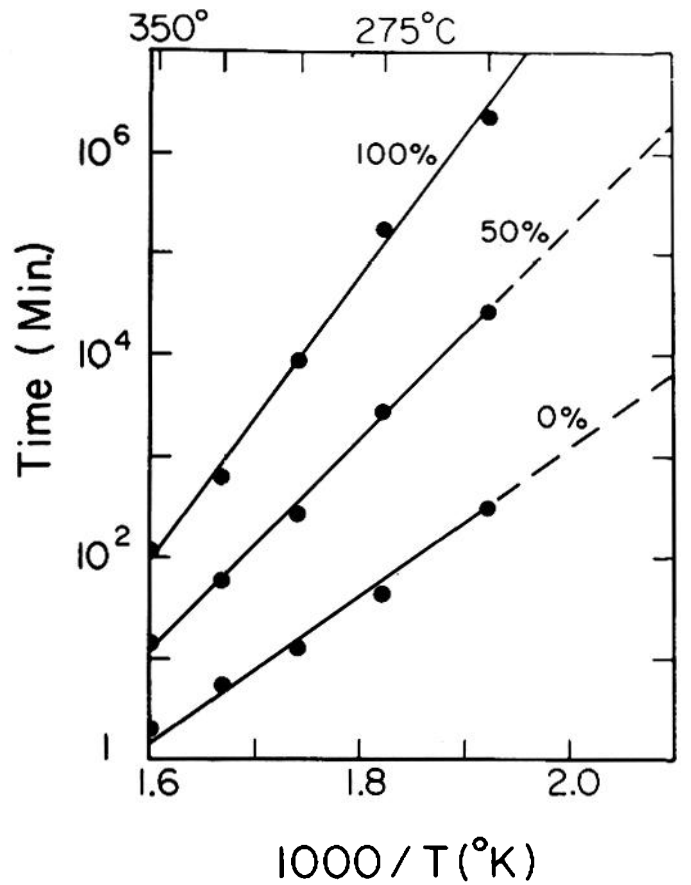

Fig. 4. - Courbes de recuit en laboratoire d'apatites d'Eldora (Colorado) extraites d'une amphibolite (d'après Naeser et Faul, 1969). Les points correspondant à un pourcentage donné d'effacement des traces sont alignés sur une droite. On constate que l'énergie d'activation pour l'effacement des traces (pente de ces droites) croît avec le taux de recuit.

(courbe $0 \%$ ). Il faut déjà plusieurs jours de recuit à cette température pour abaisser de $50 \%$ la densité de traces révélables ; enfin, pour un recuit total, une durée de quelques mois est nécessaire.

Tous les minéraux n'ont pas la même sensibilité au recuit (Fleischer et al., 1975). Ainsi, le zircon et le sphène sont beaucoup moins sensibles au recuit que par exemple des matériaux tels que l'apatite ou le verre.

\begin{tabular}{|c|c|c|c|}
\hline $\begin{array}{l}\text { POUNGEST } \\
\text { EASIYY } \\
\text { MEASURED } \\
\text { AGE (YRS) }\end{array}$ & $\begin{array}{l}\text { URANIUM } \\
\text { CONTENT } \\
\text { WT PARTS } \times 10^{-6}\end{array}$ & $\begin{array}{l}\text { YOUNGEST AGE } \\
\text { MEASURED BY } \\
\text { CONSIDERABLE } \\
\text { LABOR (YEARS) }\end{array}$ & $\begin{array}{l}\text { URAMIUM CONTENT OF } \\
\text { VARIOUS MINERALS }\end{array}$ \\
\hline $3,000 \times 10^{6}$ & 0.0001 & $80 \times 10^{6}$ & OLIVINE OUARTZ FELOS \\
\hline $300 \times 10^{6}$ & 0.001 & $8 \times 10^{6}$ & MICA \\
\hline $30 \times 10^{6}$ & 0.01 & 800,000 & \\
\hline $3 \times 10^{6}$ & 0.1 & 80,000 & GARNET \\
\hline 300,000 & 1 & 8,000 & \\
\hline 30,000 & 10 & 800 & \\
\hline 3,000 & 100 & 80 & MAGUSAL \\
\hline 300 & 1000 & 8 & DIRCOY EULOOTE \\
\hline 30 & $1 \%$ & 0.8 & \\
\hline 3 & $10 \%$ & I MONTH & nan- \\
\hline 03 & $100 \pi$ & 3 dars & GASS \\
\hline
\end{tabular}

Les énergies d'activation pour l'effacement des traces varient, entre les différentes espèces minérales étudiées (et pour différents taux de recuit) de $\sim 1$ à $10 \mathrm{eV}$ (Fleischer et al, 1975). L'extrapolation de l'équation 9

Hig. 5. - Spectre d’âges déterminables par la méthode des traces avec quelques-unes des espèces minérales less plus fréquemment utilisées, en fonction de leur teneur en uranjum. (d'après Fleischer et Price, 1964 b). 
pour les durées géologiques montrent qu'à une température $<50^{\circ} \mathrm{C}$ à peu près toutes les espèces minérales retiennent quantitativement les traces de fission.

Pour les datations à l'intérieur du quaternaire, la nécessité d'une remise à zéro du chronomètre traces de fission limite son application, d'une part aux roches volcaniques, d'autre part aux matériaux suffisamment réchauffés, c'est-à-dire où toutes les traces préexistantes ont été recuites : pierres de fours et de foyers, céramiques, roches impactées (cratères météoritiques, tectites). Parmi les phases utilisables en datations par traces de fission, seuls les verres peuvent présenter un degré de recuit notable dans les conditions naturelles, c'est-à-dire donner des âges apparents trop jeunes. L'application à ces verres, de la méthode des âges plateaux (Storzer et Poupeau, 1973) permet alors d'obtenir des âges géologiquement significatifs.

\section{Correction des âges thermiquement rajeunis}

On a constaté qu'un recuit en laboratoire affecte, pour des conditions de révélation données, non seulement la densité de traces révélables, mais aussi leur longueur celle-ci diminuant lorsque le taux de recuit des traces augmente (Fleischer et al., 1965 a). Les traces fossiles des minéraux naturels sont fréquemment plus courtes que les traces induites. L'âge apparent trace de fission de ces minéraux, est en général un âge hybride sans signification géologique. En effet, dans ce cas, les traces de fission fossile sont composées de populations présentant des taux d'effacement différents.

On peut cependant obtenir un âge traces de fission significatif si l'on tient compte de la relation entre diminution de densité révélable et réduction de longueur moyenne des traces par recuit (Storzer et Wagner, 1970). Une courbe étalon étant établie (avec des conditions de révélation constantes) pour une phase minérale donnée à partir de traces de fission induites, on déduit de la longueur moyenne des traces fossiles leur taux de recuit et donc le facteur de correction de l'âge apparent.

La méthode plus récente des âges-plateaux (Storzer et Poupeau, 1973) présente l'avantage de supprimer les mesures de longueur de traces, longues et fastidieuses. Analogue à la méthode ${ }^{39} \mathrm{Ar}-{ }^{40} \mathrm{Ar}$, elle consiste à déterminer une série d'äges traces de fission après recuits à différentes températures des traces fossiles et induites. Aux températures les plus basses, seules les traces induites sont affectées : l'âge traces de fission augmente alors avec le taux de recuit. Lorsque les traces induites parviennent au même taux de recuit que les traces naturelles, l'âge traces de fission atteint un plateau. La methode des âges-plateaux donne de bons resultats sur les verres volcaniques continentaux, La validité de cette méthode pour les verres basaltiques océaniques profonds et les minéraux n'est pas encore établie.

\section{Matériaux datables par traces de fission}

En général, toute phase minérale capable d'enregistrer les traces de fission et contenant de l'uranium est utilisable en géochronologie par traces de fission. Le choix d'une phase dépend d'une part du problème à traiter (recherche des mises en place de massifs, histoire de refroidissement ou de surrection, etc. ..) donc de ses caractéristiques de rétention des traces ; d'autre part de sa teneur en uranium, en fonction du spectre d'âges envisagé. La figure 5 présente la gamme des possibilités des espèces minérales les plus souvent utilisées. en fonction de leurs teneurs en uranium.

Dans les problèmes du quaternaire, les phases présentant les plus larges possibilités d'emploi sont les verres (fabriqués et volcaniques) et les zircons, utilisables dans certaines conditions jusqu'à moins de 1000 ans environ. On peut fréquemment y ajouter, pour le pleistocène inférieur, l'apatite et le sphène. Enfin, dans des circonstances favorables, (provinces enrichies en uranium par exemple), d'autres minéraux, comme les micas, (Bigazzi et al, 1973) peuvent s'ajouter à cette liste.

\section{Précision des âges traces de fission}

La détermination d'un âge par la méthode des traces de fission nécessite le comptage de trois densités de traces. On admet que, comme en radioactivité, chaque comptage obéit à une statistique de Poisson, c'est-à-dire que la déviation standard est donnée par:

$$
\sigma=\frac{1}{\sqrt{\mathrm{N}}}
$$

où $\mathrm{N}$ est le nombre total de traces comptées.

Un traitement simplifié des erreurs statistiques donne pour déviation standard $\sigma_{t}(\%)$ sur l'âge la valeur :

$$
\sigma_{t}=\sqrt{\sigma_{F}^{2}+\sigma_{i}^{2}+\sigma_{n}^{2}}
$$

où $\sigma_{F}, \sigma_{i}$ et $\sigma_{\mathrm{n}}$ sont les erreurs statistiques sur $\rho_{\mathrm{F}}, \rho_{\mathrm{i}}$, et $\rho_{\mathrm{n}}$ respectivement.

Lorsqu'un nombre suffisant de traces peut être compté, l'erreur statistique peut être limitée à moins de $5 \%$. Dans un traitement plus raffiné, tenant compte des relations entre les variables $\rho_{\mathrm{i}}$ et $\rho_{\mathrm{F}}$, Mc Gee et al (1976) suggèrent que l'erreur statistique peut en fait être réduite à \pm 1 à $2 \%$, dans certains cas.

La validité d'un âge traces de fission dépend donc du degré d'exactitude avec lequel on connait les constantes figurant dans l'expression de A de l'équation 4, plus difficile à évaluer, en fonction surtout de l'incertitude sur $\lambda_{F}$. Dans le calcul d'un âge à partir de l'équation (8), on peut estimer que la précision absolue sur l'âge peut être de l'ordre de \pm 6 à $8 \%$.

Deux remarques pour terminer. Tout d'abord, ces évaluations n'incluent pas l'erreur éventuellement intro- 
duite dans la correction des âges thermiquement rajeunis. Ensuite, dans le calcul des erreurs statistiques n'entrent pas les erreurs dues à l'identification des traces. Ces dernières peuvent prendre un rôle prépondérant pour les âges très jeunes.

Ce dernier point est illustré par les données réunies dans la table 1. Il s'agit de la datation de basaltes oécaniques profonds à partir du verre qui forme la bordure extérieure de pillow-lavas. Pour chacun de ces âges, entre 2 et 4 traces fossiles seulement ont été comptées pour une surface observée au microscope de l'ordre de $10 \mathrm{~cm}^{2}$. De tels âges sont très difficiles à obtenir. II requièrent des journées de travail au microscope. L'erreur statique $(2 \sigma)$ reportée dans la dernière colonne est déjà très importante, de l'ordre de $100 \%$. Mais elle n'inclut pas les erreurs éventuelles d'identification (voir Fig. 2a) qui sont tout à fait critiques dans ce cas du fait du faible nombre de traces fossiles observées.

\section{III. - EXEMPLES D'APPLICATIONS AUX PROBLEMES DU QUATERNAIRE}

\section{Chronologie absolue du quaternaire}

Ainsi que nous l'avons précisé plus haut, les seuls matériaux naturels datables par traces de fission dans le quatemaire, à l'exception des matériaux impactés, sont les roches volcaniques et les matériaux remaniés qui en proviennent.

Pour la datation du volcanisme continental, la méthode des traces est souvent employee concurremment avec celle du K-Ar (Izett et Naeser, 1976). Elle peut la remplacer pour des âges $<10^{6}$ ans (Carbonnel et Poupeau, 1969 ; Bigazzi et al, 1977). D'autre part, la méthode des traces s'avère particulièrement intéressante pour la datation des cendres vitreuses, où les mesures de $\mathrm{K}$-Ar ont tendance à donner des âges trop anciens ou perturbés par l'hydratation du matériau (Obradovitch, 1963, cité par Naeser et al, 1973). Dans le domaine oceanique Mc Dougall (1971) a toutefois montré que les fragments de verre volcanique des niveaux sédimentaires pouvaient donner des âges significatifs.
En dehors des applications à la datation du volcanisme récent la méthode des traces a surtout été appliquée, dans la géologie du quaternaire, à la définition de l'échelle absolue biostrati-graphique. Ainsi, en Nouvelle Zélande, Seward (1974) a daté les sous stages du pleistocène marin à l'aide de cendres vitreuses océaniques. De la même façon, en Californie la bio-stratigraphie de la base du quatemaire a pu être datée par Boelsstorff et Steineck (1975). En Italie, Ambrosetti et al (1972) et Bigazzi et al (1973) ont largement utilisé les datations par traces de fission des niveaux volcaniques continentaux aussi bien que marins pour préciser, en liaison avec les âges $\mathrm{K}$-Ar et les données du paléomagnétisme la chronologie absolue du quaternaire.

L'un des grands avantages de la methode des traces est d'offrir la possibilité de dater une formation à partir d'une quantité de matière très réduite. Cette propriété est particulièrement avantageuse lorsqu'on travaille sur des carottages, où certains niveaux peuvent renfermer du matériel volcanique récent, mais en abondance parfois très faible. La présence de rares fragments de verres volcaniques peut alors suffire pour déterminer un âge. Ce type de situation est actuellement exploité par Arias et al. (1977), en vue d'étudier les âges de rejeu de failles actives au cours du quaternaire dans la vallée du Pô.

Par ailleurs, pour les formations remaniées, la methode des traces permet, par la mesure d'âges sur des cristaux individuels, de reconnaître d'éventuelles contaminations par des matériaux d'âges différents. Ainsi, dans un tuff volcanique daté à 2,4 millions d'années par K-Ar et traces de fission sur zircons, Hurford et al (1976) ont identifie un groupe de zircons d'âges apparents traces de fission groupés entre 293 et $\mathbf{3 8 0}$ millions d'années. De façon plus générale, des déterminations systématiques d'âges traces de fission de minéraux détritiques dans les sédiments quaternaires pourraient contribuer à la définition de populations minérales et à l'identification des massifs d'origine de ces minéraux.

\section{Basaltes sous marins profonds}

Ces basaltes ne sont en général pas datables par potassium-argon en raison de la présence fréquente d'argon hérité et pour les plus récents de la faible teneur en ${ }^{40} \mathrm{Ar}$

TABLE 1

Age traces de fission de pillow lavas du rift Medio Atlantique (Aumento, 1969)

\begin{tabular}{|l|c|c|c|c|c|c|}
\hline Echantillon $\left(^{*}\right)$ & $\begin{array}{c}\text { surface } \\
\text { parcourue } \\
\left(\mathrm{cm}^{2}\right)\end{array}$ & $\begin{array}{c}\rho_{\left.\mathrm{F}^{(* *}\right)} \\
(\text { traces cm }\end{array}$ & $\begin{array}{c}\left.\rho_{\mathrm{i}}{ }^{+}\right) \\
\left(\text {traces } \mathrm{cm}^{-2}\right)\end{array}$ & $\mathrm{C}^{\mathrm{u}}(* * *)$ & $\begin{array}{c}\text { âge } \\
(\text { ans) }\end{array}$ & $\sigma(95 \%)$ \\
\hline AG-19-66-56-1 & 12.52 & $0.16( \pm 71 \%)$ & $7500( \pm 3.6 \%)$ & 0.25 & 12000 & \pm 18000 \\
AG-19-66-56-4 & 8.90 & $0.22( \pm 71 \%)$ & $11150( \pm 4.4 \%)$ & 0.38 & 13000 & \pm 18000 \\
CHAIN-43-103 & 9.99 & $0.40( \pm 50 \%)$ & $6990( \pm 3.8 \%)$ & 0.24 & 26000 & \pm 26000 \\
\hline
\end{tabular}

$\left.{ }^{*}\right)$ croûtes vitreuses de pillow lavas

(**) $\rho_{\mathrm{F}}$ et $\rho_{\mathrm{i}}=$ densités de traces de fission fossile et induite respectivement

$\left({ }^{* * *}\right)$ (") $^{*}=$ concentration en uranjum 
radiogénique formé in situ. Il est par contre possible de les dater à partir de la phase vitreuse qui constitue le facies de bordure des pillow-lavas (Fleischer et al, 1968). Les mesures d'âges traces de fission dans ces basaltes ont été appliquées à la mesure du taux d'expansion des fonds océaniques (Fleischer et al, 1968, Aumento, 1969) de part et d'autre du rift medio-atlantique, comme à la mesure de l"âge" du plancher ockanique (Melson, 1973 ; Reynolds et Barr, 1974).

Une récente étude de Macdougall (1976) montre d'une part que les âges apparents de ces verres sont presque toujours inférieurs à l' "âge" des fonds marins sur lesquels ils reposent ; d'autre part, que, dans ces cas, il n'est pas toujours possible d'obtenir des âges corrigés pour l'effacement thermique des traces qui soient en accord avec les âges estimés du fond océanique. Toutefois cet auteur conclut, à partir de mesures de la stabilité thermique des traces en laboratoire, que pour les basaltes quaternaires ( $<10^{6}$ ans) les effets d'annealing devraient être négligeables et donc les datations significatives.

\section{Hommes fossiles}

A deux reprises, une controverse sur l'âge des plus anciens hominiens d'Afrique orientale a pu être résolue par l'emploi de la méthode des traces.

En 1961, la datation par K-Ar du Bed I de la gorge d'Olduvai (Tanganyka) renfermant des traces d'activité de deux hominiens à $1,75 \pm 0,5$ millions d'années (Leakey et al, 1961) avait été accueillie avec scepticisme par les anthropologistes. Une datation par traces de fission de ponces vitreuses du même site par Fleischer et al (1965) produisit un âge de 2,0 $\pm 0,3$ millions d'années, confirmant ainsi l'âge K-Ar de Leakey et coll.
En 1976, la méthode des traces de fission devait à nouveau intervenir dans la datation d'un site à l'âge mal établi par la méthode K-Ar. Il s'agissait du tuff KBS (Kenya) contenant un ensemble d'artefacts pierreux surmontant un niveau avec un crâne humain. Deux datations de ce tuff par la méthode ${ }^{39} \mathrm{Ar}-{ }^{40} \mathrm{Ar}$ avaient donné un âge de 2,42 $\pm 0,01$ millions d'années (Fitch et al, 1976), significativement plus ancien qu'une valeur mesurée antérieurement de 1,82 $\pm 0,04$ millions d'années, publiée par Curtis et al (1975). Une datation par traces de fission de zircons devait foumir un âge de $2,44 \pm 0,08$ millions d'années, concordant avec à la fois l'âge ${ }^{39} \mathrm{Ar}-{ }^{40} \mathrm{Ar}$ et la stratigraphie paléomagnétique locale (Brock et Isaac, 1974). La méthode des traces devait en outre montrer que ce tuff renfermait des minéraux d'âge plus ancien, jusque vers quelques centaines de millions d'années (Hurford et al. 1976).

La méthode des traces apparaît donc comme une méthode de complément pour la datation des fossiles humains les plus anciens. Elle peut même devenir indispensable, lorsque l'application de la méthode $\mathrm{K}$-Ar est limitée par la jeunesse des sites ou des risques de contamination des formations à dater par des matériaux d'âge différent. Actuellement, cette méthode est donc employée en routine avec le K-Ar dans l'étude des sites renfermant des hominidés (voir par exemple Aronson et al, 1977).

\section{Archéologie}

En archéologie, la méthode des traces a été employée essentiellement pour la datation de sites d'occupation et l'étude des routes commerciales de l'obsidienne.

Pour dater les sites d'occupation, on utilise la propriété d'effacement des traces dans les matériaux suf-

TABLE 2

Exemples de sites et objets fabriqués datés par traces de fission

\begin{tabular}{|c|c|c|c|}
\hline $\begin{array}{l}\text { Age approximatif } \\
\text { (ans) }\end{array}$ & objet & phase datée & Références \\
\hline $\begin{array}{c}1000 \\
1000-2000\end{array}$ & $\begin{array}{l}\text { Pointe de flèche } \\
\text { Japon }\end{array}$ & $\begin{array}{l}\text { obsidienne d'un site } \\
\text { détruit par le feu }\end{array}$ & Watanabe et Suzuki 1969 \\
\hline $\begin{array}{c}1000-2000 \\
4000\end{array}$ & $\begin{array}{l}\text { Poterie } \\
\text { Japon }\end{array}$ & zircon dans un sable brûlé & Nishimura, 1971 \\
\hline 4000 & $\begin{array}{c}\text { couteau } \\
\text { Kenya }\end{array}$ & $\begin{array}{l}\text { obsidienne chauffée } \\
\text { dans un foyer }\end{array}$ & Fleischer et al, 1965 \\
\hline 5000 & $\begin{array}{l}\text { pointe de Javelot } \\
\text { Japon }\end{array}$ & $\begin{array}{l}\text { obsidienne d'un site } \\
\text { détruit par le feu }\end{array}$ & Watanabe et Suzuki, 1969 \\
\hline \multicolumn{4}{|l|}{ Verres fabriqués } \\
\hline 100 & $\begin{array}{l}\text { Vase } \\
\text { New England }\end{array}$ & verre riche en $U$ & Brill et al, 1964 \\
\hline 500 & $\begin{array}{l}\text { poterie } \\
\text { Japon }\end{array}$ & $"$ & Watanabe et Suzuki, 1969 \\
\hline
\end{tabular}


fisamment réchauffés (verres volcaniques, zircons) dans des foyers, ou au cours d'incendies. Divers exemples de sites ainsi datés entre 1000 et 5000 ans sont donnés dans la table 2 .

L'obsidienne était dans les temps préhistoriques l'objet d'un commerce intense. Au Japon, Suzuki (1973 et réfs. incluses) a utilisé la méthode des traces (datations et mesures de la teneur en uranium) pour identifier l'origine et les routes de diffusion d'objets archéologiques en obsidienne. En Europe, des travaux similaires ont été effectués par Durrani et al (1971) et Bigazzi et Bonadonna (1973).

Parmi les produits fabriqués, les objets en verre sont évidemment datables par la méthode des traces. La table 2 présente deux exemples de datation de verres fabriqués âgés de 100 a 500 ans seulement.

\section{Autres applications}

La méthode des traces ne permet pas seulement de dater des objets à partir des traces de fission spontanee de ${ }^{238} \mathrm{U}$. Elle peut aussi être utilisée dans ce but à partir des traces de fission induite de ${ }^{235} \mathrm{U}$. Elle est alors utilisable soit comme une technique de datation relative (les fossiles), soit comme une technique d'appoint pour une autre méthode de datation (thermoluminescence).

\section{Datations relatives.}

La fission induite de l'uranium 235 par les neutrons thermiques donne en effet la possibilité de réaliser une microanalyse de la distribution en uranium dans un matériau (Price et Walker, 1963 a ; Poupeau et al, 1973 ; Fleischer et al, 1975). Il suffit pour cela de placer contre une section polie de ce matériau un détecteur auxiliaire dépourvu lui-même d'uranium, mais capable d'enregistrer les traces de fission produites à l'intérieur du matériau à étudier. La révélation des traces dans le détecteur, après irradiation, donne une photographie de la distribution en uranium. Une précision de l'ordre de $10 \mathrm{mi}$ crons pour la localisation de l'uranium peut être obtenue.

Cette technique a été utilisée avec des os actuels et fossiles. Dans ce dernier cas, elle permet d'étudier les échanges d'uranium avec le milieu d'enfouissement de l'os, c'est-à-dire l'enrichissement en uranium avec le temps. Convenablement utilisée, cette technique permet, en principe, une mesure relative de l'âge d'enfouissement d'os extraits d'un même site (Fleischer et al, 1975 ; Fleischer, 1976).

\section{Thermoluminescence}

Les datations par thermoluminescence de céramiques et de roches se sont récemment développées (Aitken, 1974 ; Poupeau, 1977 ; Valladas, 1977). Dans cette méthode de datation, il est indispensable de connaître la distribution fine des éléments radioactifs $U, T h$ et $\mathrm{K}$ dans le matériau à dater, afin d'évaluer leurs effets relatifs dans la production de la thermoluminescence naturelle de ce matériau (Valladas, 1977). L'emploi de la méthode des traces permet d'obtenir ces données pour l'uranium (Poupeau et al, 1976 ; Valladas, 1977) et le thorium (Hair et al, 1971 ; Fleischer et al, 1975). Elle a déjà rendu possible une meilleure évaluation de la microdosimétrie dans les quartz de galets granitiques réchauffés utilisés pour dater par thermoluminescence un site d'occupation magdalénien de la région parisienne (Poupeau et al. 1976) et une coulée volcanique récente de la chaîne des Puys, dans le Massif Central (Valladas, 1977).

\section{IV. - CONCLUSION}

La méthode de datation par les traces de fission spontanée de l'uranium 238 présente donc un large domaine d'application aux problèmes du quaternaire, puisqu'elle s'applique aussi bien au traitement de problèmes géologiques (établissement d'une échelle absolue pour le quaternaire, datations d'événements magnétiques, de rejeu de failles, de massifs volcaniques) qu'aux relations terreespace (datations de tectites, d'astroblèmes) et qu'à l'évolution de l'espèce et des civilisations humaines (datations de gisements anciens d'hommes fossiles, d'objets et de sites archéologiques). Elle présente l'avantage d'une mise en oeuvre simple et peu couteuse. Ses limitations proviennent essentiellement de la nécessité de trouver des phases suffisamment riches en uranium.

D'autre part, en permettant une microradiographie fine de l'uranium par la fission induite de ${ }^{235} \mathrm{U}$, elle rend possible des datations relatives d'os fossiles et apparaît comme un complément indispensable dans les datations de roches par thermoluminescence.

Manuscrit reçu en septembre 1977

\section{REFERENCES}

Aitken M.J., 1974. - Physics and Archaelogy, Clarendon Press, Oxford, 291 p.

Ahrens T.J., Fleischer R.L., Price P.B. et Woods R.T., 1970. - Erasure of fission tracks in glasses and silicates by shock waves. Earth Planet. Sci. Letters, 8, p. 420-426.

Ambrosetti P., Azzaroli A., Bonadonna F. et Follieri M., 1972. - A scheme of pleistocene chronology for the tyrrhenian side of Central Italy. Bol. Soc. Geol. It., 91, p. 169-184. 
Arias C., Bigazzi G. et Bonadonna F.P., 1977. - Geochronology and paleomagnetic stratigraphy of a Po Valley (Italy) basin, 5 th European Colloquium of Geochronology, Cosmochronology and Isotope Geology, Pise 5-10 septembre, résumé.

Aronson J.L., Schmitt T.J., Walter R.C., Taieb M., Tiercelin J.J., Johanson D.C., Naeser C.W. et Naim A.E.M., 1977. - New Geochronologic and paleomagnetic data for the hominid-bearing Hadar For mation of Ethiopia. Nature, 267, p. 323-327.

Aumento F., 1969. - The Mid-Atlantic ridge, near $45^{\circ} \mathrm{N}$. Fission track and ferromanganese chronology. Can. J. of Earth Sciences, 6, p. 1431-1440.

Bigazzi G. et Bonadonna F., 1973. - Fission track dating of obsidian of Lipari Island. Nature, 242, p. $322-323$

Bigazzi G., Bonadonna F. et laccarino S., 1973. Geochronological hypothesis on plio-pleistocène boundary in latium region (Italy). Bol. Soc. Geol. It., 92 , p. 391422.

Bigazzi G., Bonadonna F.P. et Funiciello, 1977. Fission track chronology of Villa Senni tuff formation (Central Italy), 5th European Colloquium of Colloquium of Geochronology, Cosmochronology and Isotope Geology, Pise 5-10 semtembre, résumé.

Boellstorff J.D. et Steineck P.L., 1975. - The stratigraphic significance of fission track ages on volcanic ashes in the marine late cenozoic of southern california. Earth Planet. Sci. Letters, 27, p. 143-154.

Brill R.H., Fleischer R.L., Price P.B. et Walker R.M., 1964. - The fission track dating of man-made glass : part. 1, preliminary results. J. Glass Studies, 6, p. 151-155.

Brock A. et Isaac G.L., 1974. - Paleomagnetic stratigraphy and chronology of bearing sediments east of Lake Rudolf, Kenya. Nature, 247, p. 344-348.

Carbonnel J.P. et Poupeau G., 1969. - Premiers éléments de datation absolue par traces de fission des basaltes de l'Indochine méridionale. Earth Planet. Sci. Letters, 6, p. 26-30.

Carpenter B.S. et Reimer G.M., 1974. - Calibrated glass standards for fission track use. N.B.S. Special Publication 260-49, 16 p.

Curtis G.H., Drake R.F., Cerling T.E., Cerling B.L. et Hampel J.H., 1975. - Age of KBS tuff in Koobi Fora Formation, East Rudolf, Kenya. Nature, 258, p. 395-398.

Drozd R.U., Morgan C.J., Podosek F.A., Poupeau G., Shirck J.R. et Taylor G.J., 1977. - Plutonium244 in the early solar system. Astrophysical J., 212 , p. 567-580. Durrani S

Durrani .S., Khan H., Taj M. et Renfrew C., 1971. Obsidian source identification by fission track analysis. Nature, 233, p. 242-245.
Fitch F.J., Hooker P.J. et Miller J.A., 1976. $-{ }^{40} \mathrm{Ar}-{ }^{39} \mathrm{Ar}$ dating of the KBS Tuff in Koobi Fora Formation, East Rudolf, Kenya. Nature, 263, p. 740-744.

Fleischer R.L., 1976. - Fission tracks and Archaelogy, In Datations absolues et analyses isotopiques en préhistoire. Méthodes et limites, Colloque 1 , IX ${ }^{\text {ème }}$ Congrès de l'Union Internationale des Sciences Préhistoriques et Protohistoriques, Ed. C.N.R.S., Paris, p. 82-100.

Fleischer R.L. et Price S.B., 1964, a. - Decay constant for spontaneous fission of $\mathrm{U}^{238}$. Thys. Rev., 1336, p. 63-64.

Fleischer R.L. et Price P.B., 1964, b. - Techniques for geological dating of minerals by chemical etching of fission fragment tracks. Geochim. Cosmochim. Acta, 28, p. $1705-1714$.

Fleischer R.L., Price P.B., Symes E.M. et Miller D.S., 1964, a. - Fission track ages and track annealing beviour of some micas, Science, 143, 394-351.

Fleischer R.L., Price P.B. et Walker R.M., 1964, b. Fission track ages of zircons. J. Geophys. Res., 69, p. 4885-4888.

Fleischer R.L., Price P.B. et Walker R.M., 1965, a. Effects of temperature, pressure and ionisation on the formation of fission tracks in minerals and glasses. J. Geophys. Res., 70, p. 1497-1502.

Fleischer R.L., Price P.B. et Walker R.M., 1965, b. Neutron flux measurements by fission tracks in solids. Nucl. Sci. Eng., 22, p. 153-156.

Fleischer R.L., Price P.B., Walker R.M. et Leakey L.B.S., 1965 c. - Fission track dating of Bed I, Olduvai Gorge. Science, 148, p. $72-74$.

Fleischer R.L., Price P.B., Walker R.M. et Leakey L.B.S., 1965, d. - Fission track dating of a Mesolithic Knife. Nature, 205, p. 1138

Fleischer R.L., Price P.B. et Walker R.M., 1965, e. The ion explosion spike mechanism for formation of charged particle tracks in solids. J. Appl. Phys. 36, p. $3645-3652$.

Fleischer R.L., Viertl J.R.M., Price P.B. et Aumento F., 1971. - A chronological test of ocean bettom spreading in the North Atlantic. Rad. Effect, 11, p. 193194.

Fleischer R.L. et Hart H.R., 1972. - Fission track dating : techniques and problems, in "Calibration of hominoid evolution", Ed. W.W. Bishop and A. Miller, University of Toronto Press, p. 135-170.

Fleischer R.L., Woods R.T., Hart H.R., Price P.B. et Short, N.M. 1974. - Effects of schoks on fission track dating of apatite and sphène crystals from the Hardhat and Sedan underground nuclear explosions. J. Geophys. Res., 79, p. 339-342. 
Fleischer R.L., Price P.B. et Walker R.M., 1975. Nuclear tracks in Solids, University of California Press, 605 p.

Galliker D., Hugentobler E. et Hahn, 1970. - Spontane Kenspaltung von ${ }^{238} \mathrm{U}$ und ${ }^{241} \mathrm{Am}$. Helv. Phys. Acta, 43, p. 593-606.

Gerling E.K., Shukolyukov Y.A. et Makarochkin B.A., 1959. - Determination of the half-life of the spontaneous uranium-238 desintegration from the xenon content in uranium minerals. Radiokhimiya, 1 , p. 223-226.

Gleadow A.J.W. et Lowering J.F., 1974. - The effects of weathering on fission track dating. Earth Planet. Sci Letters, 22, p. 163-168.

Hair M.W., Kaufhold J., Maurette M. et Walker R.M., Hair M.W., Kaufhold J., Maurette M. et Walker R.M., 9 1971. - Th Microanalysis using fission track dating. Rad. Effects, 7, p. 285-287.

Hurford A.J., Gleadow A.J.W. et Naeser C.W., 1976. Fission-track dating of pumice from the KBS Tuff, East Rudolf, Kenya. Nature, 263, p. 738-740.

Hurford A.J. et Gleadow A.J.W., 1977. - Galibration of fission track dating parameters, Nuclear Track Detection, 1, p. 41.48:

Izett G.A. et Naeser C.W., 1976. - Age of the Bishop Tuff of eastern California as determined by the fission-track method. Geology, 4, p. 587-590.

Lakatos S. et Miller D.S., 1970. - Water-pressure effect on fission track annealing in an alpine muscovite. Earth Planet. Sci. Letters, 9, p. 77-81.

Lakatos S. et Miller D.S., 1972. - Evidence for the effect of water content on fission track annealing in volcanic glass. Earth Planet. Sci. Letters, 14, p. $128-130$.

Leakey L.B.S., Evernden J.F. et Curtis G.H., 1961. Age of Bed I, Olduvai Gorge, Tanganika, Nature, 191 , p. 478-479.

Macdougall D., 1971. - Fission track dating of volcanic glass shards in marine sediments. Earth Planet. Sci. Letters, 10 , p. $403-406$.

Macdougall J.D., 1976. - Fission track annealing and correction procedures for oceaninc basalt glasses. Earth Planet. Sc. Letters, 30, p. 19-26.

McGee V.E., Johnson N.M. et Naeser C.W., 1976. Statistical treatment of experimental errors in the fission track dating method, Preprint.

Melson W.G., 1973. - Basaltic glasses from the Deep Sea Drilling Project : chemical characteristics, compositions of alteration products and fission track ages. Trans. Amer. Geophys. Union, 54, p. 10111014.
Maurette M., Pellas P. et Walker R.M., 1964. - Etude des traces de fission dans le mica. Bull. Soc. Fr. Miner. Crist., 87, 6-17.

Naeser C.W. et Faul H., 1969. - Fission track annealing in apatite and sphene. $J$. Geophys. Res, 74, p. 705710.

Naeser C.W., Izett G.A. et Wilcox R.E., 1973. - Zircon fission track ages of Pearlette family ash beds in Meade County, Kansas. Geology, 1, p. 187-189.

Naeser C.W. et Fleischer R.L., 1975. - The age of apatite at Cerro de Mercado, Mexico : a problem for fission track annealing corrections. Geophys. Res. Letters, 2, p. 67-70. Nishimura

Nishimura S., 1971. - Fission track dating of archaeological materials from Japan. Nature, 230 , p. 242 243.

Petit J.C., 1977. - Contribution de l'étude des dégâts d'irradiation à la compréhension du phénomène d'Oklo, Thèse $3^{\text {ème }}$ Cycle, Université Paris VII.

Pertzhak K.A. et Flerov G.N., 1940. - Spontaneous fission of uranium. Phys. Rev., 58, p. 89

Poupeau G., 1977. - Les datations par thermoluminescence en archéologie, dans Séminaire $1977 \mathrm{du}$ Pr. A. Leroi-Gourhan, Collège de France, sous presse.

Poupeau G., Selo M., Gaven C. et Cheminee J.L., 1973. Répartition de l'uranium sur la méthode des traces de fission dans la série volcanique de l'Erta'Ale (Afar). C.R. Acad. Sci., Paris, D 278, p. 405-409.

Poupeau G., Sutton S., Walker R.M. et Zimmermann D.W., 1976. - Thermoluminescent dating of fired rocks : Application to the site of Pincevent (france), Congrès de l'Union Internationale des Sciences Préhistoriques et Protohistoriques, Section Méthodologie, sous presse.

Price P.B. and Walker R.M., 1962, a. - Observation of fossil particle tracks in natural micas. Nature, 196, p. $732-734$.

Price P.B. et Walker R.M., 1962, b. - Chemical etching of charged particle tracks. J. Appl. Phys., 33, p. 3407 3412

Price P.B. et Walker R.M., 1963 a. - A simple method of measuring low uranium concentrations in natural crystals. Appl. Phys. Letters, 2, p. 23-25.

Price P.B. and Walker R.M., 1963 b. - Fossil tracks of charged particle in mica and the age of minerals. J. Geophys. Res., 68, p. 4847-4862.

Reynolds P.H. et Barr S.M., 1974. - DSDP specimens 26-251 A - 29-1 : K-Ar and fission track fating, Initial Reports of the Deep Sea Drilling Project, U.S. Gouvernement Printing Office, Washington D.C., 26, p. 515-516. 
Roberts J.H., Gold R. et Armani R.J., 1968. - Spontaneous fission decay constant of ${ }^{238} \mathrm{U}$. Phys. Rev., 174 , p. 1482-1484.

Schreurs J.W.H., Friedman A.M., Rokop D.J., Hair M.W. et Walker, 1971. - Calibrated U-Th glasses for neutron dosimetry and determination of Uranium and Thorium concentrations by the fission track method. Rad. Effects, 7, p. 231-233.

Seward D., 1974. - Age of New Zealand pleistocène substages by fission track dating of glass shards from tephra horizons. Earth Planet. Sci. Letters, 24, p. 242-248.

Shukoljikov J., Kirsten T. et Jessberger E.L., 1974. The Xe-Xe spectrum technique, a new dating method, Earth Planet. Sci. Letters 24, p. 271-281.

Steven T A., Mehnert H.H. et Obradovitch J.D., 1967. Age of volcanic activity in the San Juan Mountains, Colorado, U.S. Geol. Surv. Prof. Paper, 575-D, p. 47-55.

Storzer D., 1970. - Spaltspuren des 238-Urans und irhe be denting für die geologische naturlichen gläser, Thèse, Heidelberg.

Storzer D. et Wagner G.A., 1970. - A correction method for thermally lowered fission track ages. Rad. Effects, 5, p. 129-131.

Storzer D. et Poupeau G., 1973. - Ages-plateaux de minéraux et verres par la méthode des traces de fission. C.R. Acad. Sci. Paris, 276, p. 137-139.

Storzer D. et Poupeau G., 1973. - Constante de décroissance de fission spontanée de ${ }^{238} \mathrm{U}$ et datation par traces de fission. Réunion Ann. Sci. Terre, Ed. Soc. Géol. Fr., p. 387

Suzuki M., 1973. - Chronology of prehistoric human activity in Kanto, Japan part I, p. 242-328 ; part II, p. 396-469.

Teitsma A., Clarke W.B. et Allegre C.J., 1975. - Spontaneous fission-neutron fission xenon : a new technique for dating geological events, Science, 189, p. 878-880.

Thiel K. et Herr W., 1976. - The ${ }^{238} U$ spontaneous fission decay constant re-determined by fission tracks. Earth Planet. Sci. Letters, 30, p. 50-56.

Valladas G., 1977. - La datation des roches par la thermoluminescence. Application à une coulée volcanique. Bull. Assoc. Fr. pour l'Etude du Quaternaire, sous presse.

Wagner G.A., Reimer G.M., Carpenter B.S., Faul H., Van der Linden R. et Gijbels R., 1975. - The spontaneous fission rates of U-238 and fission tracks, Geochim. Cosmochim. Acta, 39.

Wagner G.A., Storzer D. et Keller J., 1976. - Fission track dating of quaternary volcanic glasses from the Mediterranean. N. Jb. Miner. Mhe, H2, p. 84-94.

Walker R.M., 1963. - Characteristics and applications of solid state track detectors, Proc. Strasbourg Conf. on New Methods of track detection, Centre de Recherches Nucléaires, Strasbourg.

Watanabe N. et Suzuki M., 1969. - Fission track dating of archaelogical glass materials from Japan. Nature, 222 , p. 1057-1058. 